\section{General medicine and surgery for dental practitioners. Part 2 - metabolic disorders}

\author{
M. Greenwood ${ }^{1}$ and J. G. Meechan ${ }^{2}$
}

IN BRIEF

- Metabolic disorders, or drugs which affect metabolism, are commonly seen in dental patients.

- There will often be no physical signs in the patient with metabolic problems.

- A thorough history is particularly important and a thorough family history will often uncover important findings.

- Knowledge of metabolic disorders will highlight patients who need special precautions to facilitate safe treatment.

It is important for dental practitioners to have a basic knowledge of the more common metabolic disorders as some may impact on the practice of dentistry. Many of these disorders do not have overt clinical signs. Taking a thorough medical history and where necessary, liaising with the patient's physician, is particularly important.

\section{INTRODUCTION}

Knowledge of metabolic disorders is essential for the safe management of dental patients. This paper considers conditions that impact on the practice of dentistry.

\section{POINTS IN THE HISTORY}

Several points in the history may reveal an underlying metabolic disorder (Table 1).

\section{Hypercholesterolaemia}

Hypercholesterolaemia is a condition which has received significant attention in recent years. It has little significance for the provision of local anaesthesia but the possibility of ischaemic heart disease

\section{GENERAL MEDICINE AND SURGERY FOR DENTAL PRACTITIONERS}
1. The older patient
2. Metabolic disorders
3. Skin disorders (A)
4. Skin disorders (B)
5. Psychiatry
6. Cancer, radiotherapy and chemotherapy

should be remembered. Low density lipoproteins (LDL) are associated with a higher risk of coronary heart disease. They are formed from intermediate density lipoproteins in the liver and contain a core of cholesterol. They bind to LDL receptors and are taken up by cells. This is particularly so when there is inhibition of 3-hydroxy-3methylglutaryl coenzyme A reductase (HMG CoA reductase), which raises cellular cholesterol. On the other hand, high density lipoproteins (HDL) are associated with a reduced risk of coronary heart disease. They carry cholesterol back to the liver from intercellular tissue.

\section{Porphyrias}

Porphyrias are rare disorders but potentially impact on dental treatment. They arise principally as the result of errors in haem metabolism causing accumulation of porphyrins, which are intermediate compounds in haemoglobin synthesis. Two main groups are recognised. These are the liver (hepatic) porphyrias and the red blood cell (erythropoietic) porphyrias. Patients with porphyrias may remain asymptomatic, but acute illness or drugs may precipitate an attack.

Variegate porphyria is the commonest form and is found mainly in people of Afrikaans descent. Acute intermittent porphyria affects all populations but is seen less frequently. A further form, hereditary coproporphyria is also recognised.

Between attacks a patient with porphyria may appear normal although

\begin{tabular}{l}
$\begin{array}{l}\text { Table } 1 \text { Points in the history in patients } \\
\text { with metabolic disorders }\end{array}$ \\
\hline Hypercholesterolaemia \\
\hline Porphyria (or subtypes) \\
\hline Malignant hyperpyrexia \\
\hline Neuroleptic malignant syndrome \\
\hline G6PD deficiency \\
\hline Suxamethonium apnoea \\
\hline Haemochromatosis \\
\hline Amyloidosis \\
\hline Abnormalities of carbohydrate metabolism \\
\hline
\end{tabular}

they may have photosensitive skin resulting in rashes. An acute attack may result in neuropsychiatric symptoms. Porphyria that becomes acute as a result of drugs may cause cardiovascular symptoms such as hypertension and tachycardia. Gastrointestinal symptoms may also occur. Some of the drugs that dentists prescribe can induce an acute attack of porphyria. Details of these are given later.

\section{Malignant hyperpyrexia}

Malignant hyperpyrexia (MH) is a rare but potentially fatal condition. It is inherited and presents as a rapid temperature rise if the patient is subjected to general anaesthesia or other medications. The family history may elicit a history of MH. Two forms are recognised: firstly, an autosomal dominant type where patients are normal between attacks, and secondly, a recessive form that affects 
boys with muscle disorders, for example myotonic dystrophy.

The most common trigger of $\mathrm{MH}$ is a combination of halothane ${ }^{1}$ (an anaesthetic agent) and muscle relaxants including suxamethonium. ${ }^{2}$ The rising temperature is accompanied by tachycardia or arrhythmias and hypotension. Management involves removing the precipitating cause, cooling and hyperventilating the patient to correct any respiratory acidosis and the use of dantrolene.

\section{Neuroleptic malignant syndrome}

Neuroleptic malignant syndrome (NMS) is rare but again potentially fatal. It is induced by the administration of certain drugs, but none commonly used in dentistry. It is recognised clinically by disturbance in the patient's mental state, autonomic function and temperature regulation.

\section{Glucose-6-phosphate dehydrogenase deficiency}

Patients may give a history of known glucose-6-phosphate dehydrogenase (G6PD) deficiency. This is the most common enzyme deficiency seen mainly in people of Mediterranean, African, Asian or Middle Eastern descent. Glucose can be metabolised via the glycolytic or the hexose monophosphate shunt pathways (Fig. 1). G6PD is involved in the latter. A derivative of the hexose monophosphate shunt is NADPH, which is involved in removing dangerous oxidative metabolites. If the activity of G6PD is low, methaemoglobinaemia (oxidised haemoglobin molecule) results and when red cells are exposed to oxidising agents, sometimes present in drugs, haemolysis results. Diagnosis is confirmed by measuring levels of the enzyme. The haemolysis is usually self-limiting but occasionally splenectomy is required.

\section{Suxamethonium apnoea}

Sensitivity to suxamethonium or scoline ('scoline apnoea') is sometimes seen and patients are often aware of its existence in the family. It is inherited as an autosomal recessive trait. Suxamethonium is a muscle relaxant that acts as a depolarising neuromuscular blocker. It behaves in a similar way to acetylcholine at the neuromuscular junction. Its action is usually brief since it is quickly destroyed by plasma cholinesterase. Around 1 in 2,000 of the population has a defect in plasma cholinesterase. This makes such people abnormally sensitive when suxamethonium is administered, resulting in persistent muscle paralysis. This means that the patients are unable to breathe for themselves (apnoea). It is postulated that the disorder may be a form of hypersensitivity reaction.

\section{Haemochromatosis}

Patients may give a history of haemochromatosis. This is a disorder of iron metabolism resulting in too much iron being absorbed from the intestine, which causes high serum ferritin levels. Deposition of iron occurs in the liver, joints, skin, adrenal glands and various other organs including the heart. The iron is deposited as haemosiderin. This is more problematic in males than females due to menstrual blood loss. The haemosiderin deposits provoke a fibrotic reaction that can produce disorders wherever the deposits are found, resulting in disorders such as cirrhosis, adrenocortical insufficiency, cardiomyopathy, skin pigmentation and diabetes (socalled 'bronze' diabetes). The condition is treated by blood letting to reduce the iron load and sometimes medically using the chelating agent desferrioxamine.

\section{Amyloidosis}

Amyloidosis (Fig. 2) is a disorder characterised by the deposition of eosinophilic hyaline protein in the tissues. It is really a manifestation of disease processes. It can affect the functions of the heart, gastrointestinal tract, spleen, kidney, liver and adrenals when it is deposited there. There may also be a bleeding tendency due to a Factor X defect.

\section{Abnormalities of carbohydrate metabolism}

Defects in carbohydrate metabolism are uncommon but may be seen in dental patients. The two main conditions are glycogen storage diseases and defects in fructose metabolism. In the glycogen storage disorders there is accumulation of the polysaccharide as a result of inherited defects in the enzymes that metabolise it. This causes hypoglycaemia. The glycogen accumulates preferentially in liver and muscle resulting in an enlarged liver, muscle pain and weakness (including the respiratory muscles). Heart failure

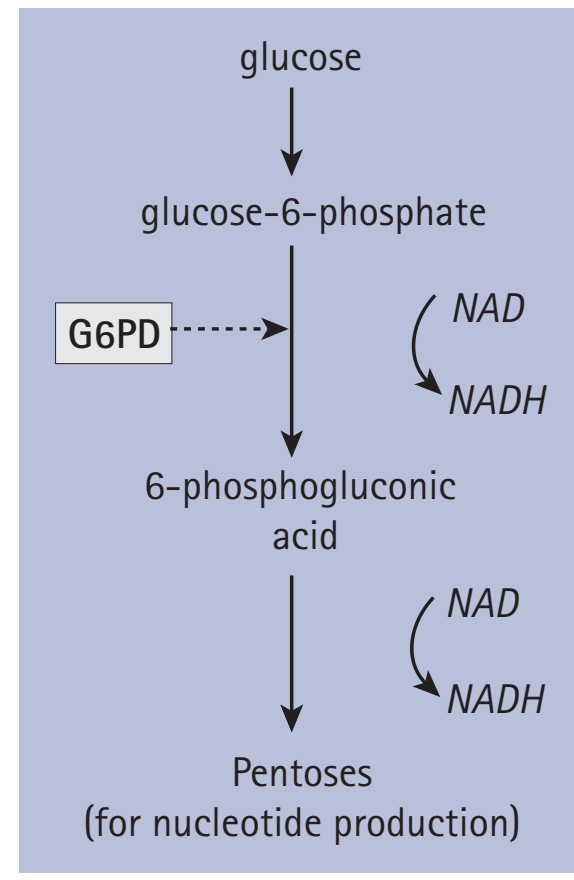

Fig. 1 The role of glucose-6-phosphate dehydrogenase (G6PD) in the hexose monophosphate shunt pathway

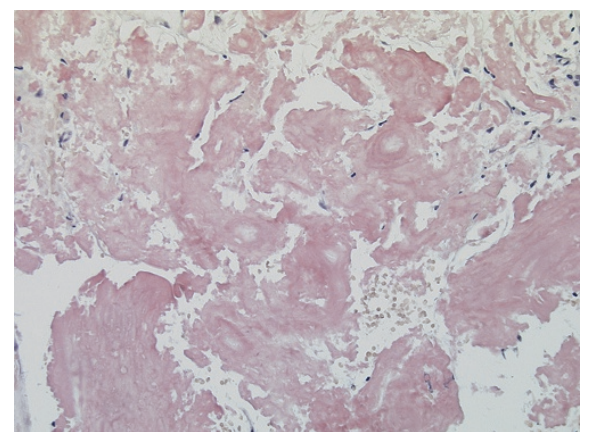

Fig. 2 The histological appearance of amyloid stained with Congo Red

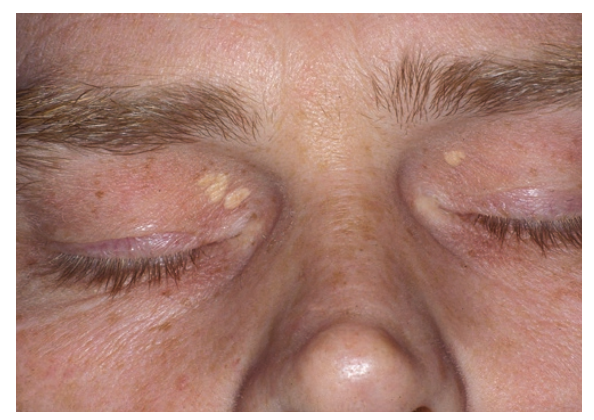

Fig. 3 Xanthelasmas in the eyelids

may also supervene. One subdivision of glycogen storage disease produces a bleeding tendency.

\section{EXAMINATION OF THE DENTAL PATIENT WITH A METABOLIC DISORDER}

There is often little to be seen in patients with metabolic disorders that would facilitate diagnosis of their condition. Some 
observations which may give a clue to an underlying metabolic condition are discussed below.

Xanthomas are yellowish plaques that may be apparent on the skin. These are termed xanthelasmas when on the eyelids (Fig. 3). They are seen in familial hyperlipidaemia and are associated with accelerated atherosclerosis and coronary heart disease.

In haemochromatosis, the skin may take on a bronze hue. Patients with porphyria may be subject to photosensitive skin rashes.

Amyloidosis may only be diagnosed formally by biopsy. It can present with an intraoral lesion, for example deposition in the tongue may lead to enlargement or localised swelling. The gingivae may also be affected. Bullae and petechiae may be seen intra-orally.

\section{DENTAL MANAGEMENT OF PATIENTS WITH METABOLIC DISORDERS}

It should be remembered in the patient with hypercholesterolaemia that there is an increased risk of ischaemic heart disease. This heightens the risks of general anaesthesia. Conscious sedation is a suitable alternative but local anaesthesia is the preferred method.

The safest management of a patient with porphyria is care with drug prescription. This is discussed below. It should be borne in mind, however, that data in this area are still incomplete. Thus the prescription of drugs in this group of patients should be carried out with this in mind.

Some drugs should be avoided in cases with G6PD deficiency for fear of producing haemolysis. The only drugs that may be used in the practice of dentistry that fall into this category are the sulphonamide antibacterial drugs.

In patients with malignant hyperpyrexia $(\mathrm{MH})$, it is important to treat infections promptly and aggressively as they are known to be potential precipitants of an attack. It is safe to give local anaesthetics. The response to adrenaline (epinephrine) may be similar to the early signs of an $\mathrm{MH}$ reaction and this should be remembered. Such signs include tachycardia, rising blood pressure and rapid breathing. Adrenaline also potentiates the release of calcium in muscle cells, which theoretically would enhance $\mathrm{MH}$; because of this dose limitation is important. The use of sedation in patients with $\mathrm{MH}$ is discussed below.

In patients with suxamethonium sensitivity, a careful history will usually uncover this; however some patients may be unaware of the condition. The use of local anaesthetics in such cases is discussed below.

Drugs that may be used in the practice of dentistry that are considered unsafe in patients with porphyria include tricyclic and monoamine anti-depressants, sulphonamides, anticonvulsant medications such as carbamazepine and phenytoin, diazepam, chloral hydrate and triclofos, clindamycin, doxycycline, erythromycin, ketoconazole, metronidazole, miconazole and oxytetracycline.

\section{Factors affecting dental treatment under local anaesthesia, sedation, general anaesthesia and management in dental practice}

The first step in management is to obtain a comprehensive history, which will alert the practitioner to the patient suffering from a metabolic disorder. Many of these patients will have no overt clinical signs.

The patient with hypercholesterolaemia is best treated under local anaesthesia, but conscious sedation is a suitable mode of treatment. The risk of ischaemic heart disease should be remembered with general anaesthesia.

In the patient with porphyria, consideration needs to be given in the use of local anaesthesia as sodium metabisulphite, which is contained in some local anaesthetic preparations to prevent the oxidation of adrenaline, can precipitate an attack. ${ }^{3}$ Lidocaine and prilocaine are considered safe when administered for local anaesthesia.

Local anaesthesia is considered safe in patients who give a history of malignant hyperpyrexia. ${ }^{4}$ As stated earlier, however, adrenaline can produce similar signs to the early stages of $\mathrm{MH}$ and this should be remembered. It is therefore wise to limit the dose of adrenaline in these patients. Adrenaline should not be used as a topical agent to manage gingival bleeding in such patients.

Benzodiazepines are not considered to be triggers of $\mathrm{MH}$ and conscious sedation using inhalation sedation is usually safe. ${ }^{4} \mathrm{MH}$ has, however, been reported after inhalation of nitrous oxide ${ }^{5}$ but this is very rare.

In patients with G6PD deficiency, local anaesthesia may induce methaemoglobinaemia in high doses. The most likely candidates are prilocaine and the topical agent benzocaine. ${ }^{6}$ Inhalation sedation is usually safe. General anaesthesia should be given in a hospital environment. The use of large doses of aspirin and the sulphonamide antibacterial drugs may precipitate haemolysis in patients with G6PD deficiency and should be avoided.

Patients with suxamethonium sensitivity may metabolise local anaesthetics differently from normal. As mentioned above this condition results from defects in plasma esterases. All of the injectable local anaesthetics used in modern dental practice are amides, unlike the earlier ester procaine. Nevertheless one of the amides, articaine, is initially metabolised in plasma by esterases ${ }^{7}$ so the use of this drug in patients with suxamethonium sensitivity may increase the toxicity of the anaesthetic. If a patient's history reveals sensitivity to suxamethonium they can be safely treated under general anaesthetic with avoidance of this muscle relaxant.

Haemochromatosis affects dental management in many ways, although local anaesthesia is usually safe. The possibility of cirrhosis, cardiomyopathy, diabetes and adrenocortical insufficiency should be borne in mind in these patients. Providing that liver function is adequate, conscious sedation is usually safe.

Local anaesthesia and conscious sedation can safely be given in patients with amyloidosis in most cases. It should be remembered, however, that amyloid can affect diverse organ systems. If the heart, adrenal glands or kidneys are involved or a bleeding tendency has developed, this will affect function and/or haemostasis.

Methaemoglobinaemia is a conversion of iron in haemoglobin from the ferrous to the ferric form. This latter configuration of iron does not allow such good availability of oxygen to the tissues which can result in cyanosis. Methaemoglobinaemia is a side effect of injecting large doses of some local anaesthetics, especially prilocaine $^{8}$ and articaine. Thus if using these drugs dose limitations should be employed or an alternative local anaesthetic should be used in the anaemic patient. 


\section{Effects of drugs used in patients with metabolic disorders on dental management}

Some of the lipid-regulating drugs can increase bleeding after surgery. The anion-exchange resins colestyramine and colestipol may interfere with the absorption of vitamin $\mathrm{K}$ leading to hypoprothrombinaemia. Ezetimibe, which interferes with the absorption of cholesterol, may rarely cause a reduction in platelet numbers. The fibrates, such as bezafibrate, may also cause a thrombocytopaenia as well as reducing white cell numbers and causing anaemia.

Statins such as atorvastatin can also produce a thrombocytopaenia. Thrombocytopaenia with a platelet count of less than $50 \times 10^{9} /$ litre precludes elective oral surgery; when levels are less than $100 \times 10^{9} /$ litre, tooth sockets should be packed with a haemostatic agent and sutured. Omega-3-acid ethyl esters can occasionally produce taste disturbance.

A number of drugs may be prescribed in the management of amyloidosis. The use of cytotoxic drugs and corticosteroids such as dexamethasone can lead to opportunistic oral infections, delayed healing and poor haemostasis. Colchicine, which is sometimes used in symptomatic treatment, can produce stomatitis and glossitis.

\section{Drug interactions with drugs used in dentistry}

One of the unwanted effects of statins is myopathy. The incidence of this is increased when statins are administered concurrently with other drugs, some of which may be prescribed to treat orofacial conditions. The important drugs in this regard are the antibacterial erythromycin and the azole antifungals. Erythromycin, itraconazole, ketoconazole and miconazole should not be prescribed to patients receiving simvastatin. Similarly, itraconazole should be avoided in patients taking atorvastatin. The toxicity of colchicine is increased during concurrent therapy with erythromycin.

\section{CONCLUSIONS}

Metabolic disorders can impact on dental treatment in diverse ways. A thorough history and a high index of suspicion are essential for safe patient management.

1. Fukami M C, Ganzberg SI. A case report of malignant hyperthermia in a dental clinic operating room. Anesth Prog 2005; 52: 24-28.

2. Monaghan A, Hindle I. Malignant hyperpyrexia in oral surgery - case report and literature review. Br J Oral Maxillofac Surg 1994; 32: 190-193.

3. Brown G J, Welbury R R. Porphyria in dental practice. Br Dent J 2002; 193: 145-146.

4. Malignant Hyperthermia Association of the United States. Malignant hyperthermia. A concern in dentistry and oral and maxillofacial surgery. Sherburne, NY: MHAUS, 2002. http://www.mhaus. org/index.cfm/fuseaction/OnlineBrochures. Display/BrochurePK/ABD1DA74-4433-48F3A4C7902B67F6FCFB.cfm

5. Ellis F R, Clarke I M C, Appleyard T N, Dinsdale R C W. Malignant hyperthermia induced by nitrous oxide and treated with dexamethasone. Br Med $J$ 1974; 4: 270-271.

6. Coleman M D, Coleman N A. Drug-induced methaemoglobinaemia. Treatment issues. Drug Saf 1996; 14: 394-405.

7. Oertel R, Rahn R, Kirch W. Clinical pharmacokinetics of articaine. Clin Pharmacokinet 1997: 33: 417-245.

8. Adams V, Marley J, McCarroll C. Prilocaine induced methaemoglobinaemia in a medically compromised patient. Was this an inevitable consequence of the dose administered? Br Dent J 2007 203: 585-587. 